\title{
THE
}

\section{The Effects of Concentric Ring Electrode Electrical Stimulation on Rat Skin}

Walter Besio

University of Rhode Island, besio@uri.edu

V. Sharma

J. Spaulding

Follow this and additional works at: https://digitalcommons.uri.edu/ele_facpubs

The University of Rhode Island Faculty have made this article openly available.

Please let us know how Open Access to this research benefits you.

This is a pre-publication author manuscript of the final, published article.

Terms of Use

This article is made available under the terms and conditions applicable towards Open Access Policy Articles, as set forth in our Terms of Use.

\section{Citation/Publisher Attribution}

Besio, W., Sharma, V. \& Spaulding, J. Ann Biomed Eng (2010) 38: 1111. https://doi.org/10.1007/ s10439-009-9891-y

Available at: https://doi.org/10.1007/s10439-009-9891-y

This Article is brought to you for free and open access by the Department of Electrical, Computer, and Biomedical Engineering at DigitalCommons@URI. It has been accepted for inclusion in Department of Electrical, Computer, and Biomedical Engineering Faculty Publications by an authorized administrator of DigitalCommons@URI. For more information, please contact digitalcommons-group@uri.edu. 


\title{
The Effects of Concentric Ring Electrode Electrical Stimulation on Rat Skin
}

\author{
W. Besio ${ }^{1}$, V. Sharma ${ }^{2}$, and J. Spaulding ${ }^{3}$ \\ ${ }^{1}$ Electrical, Computer, and Biomedical Engineering Department, University of Rhode Island, 4 East \\ Alumni Avenue, Kingston, RI 02881, USA \\ ${ }^{2}$ Huntington Medical Research Institute, Pasadena, CA, USA \\ ${ }^{3}$ Biological Services Department, Louisiana Tech University, Ruston, LA, USA
}

\begin{abstract}
Surface electrodes are commonly used electrodes clinically, in applications such as functional electrical stimulation for the restoration of motor functions, pain relief, transcutaneous electrical nerve stimulation, electrocardiographic monitoring, defibrillation, surface cardiac pacing, and advanced drug delivery systems. Common to these applications are occasional reports of pain, tissue damage, rash, or burns on the skin at the point where electrodes are placed. In this study, we quantitatively analyzed the effects of acute noninvasive electrical stimulation from concentric ring electrodes (CRE) to determine the maximum safe current limit. We developed a three-dimensional multi-layer model and calculated the temperature profile under the CRE and the corresponding energy density with electrical-thermal coupled field analysis. Infrared thermography was used to measure skin temperature during electrical stimulation to verify the computer simulations. We also performed histological analysis to study cell morphology and characterize any resulting tissue damage. The simulation results are accurate for low energy density distributions. It can also be concluded that as long as the specified energy density applied is kept below $0.92\left(\mathrm{~A}^{2} / \mathrm{cm}^{4} \cdot \mathrm{s}^{-1}\right)$, the maximum temperature will remain within the safe limits. Future work should focus on the effects of the electrode paste.
\end{abstract}

\section{Keywords}

Noninvasive electrical stimulation; Transcutaneous electrical stimulation; Electrical-thermal coupled field analysis; Infrared thermography

\section{INTRODUCTION}

Surface electrodes are the most commonly used electrodes clinically, in applications as diverse as functional electrical stimulation for the restoration of motor functions, pain relief, transcutaneous electrical nerve stimulation, defibrillation, surface cardiac pacing, and advanced drug delivery systems. ${ }^{27}$ Common to these applications are occasional reports of pain, tissue damage, erythema, rash, or burns on the skin at the point where electrodes are placed. . $^{1,11,18,19,24,28,30,31,33}$ The origin of these electrical burns is not well understood. Heat development under the electrode during the passage of electrical current has been reported as 
a major cause of skin burn. $3,8,14,28,33$ Temperature elevation of skin above $45{ }^{\circ} \mathrm{C}$ has been reported to result in tissue damage even from short-term exposure. ${ }^{22,28}$ Takamiya et al. $^{32}$ found that electrical energy generates joule heat. Thus, this phenomena in electrical injuries was caused by the effect of heat. In a review by Merrill et al.,20 although mainly for implantable stimulation techniques, several possible mechanisms of injury are discussed which are still pertinent for noninvasive electrical stimulation. A common factor contributing to all the above mentioned mechanisms are regions of high current density. ${ }^{13,17,22,26,27}$ Overmyer et al. ${ }^{24}$ modeled this phenomenon and found from experimental observations that the area of the highest temperature increase, or burns, was usually at the edge of the electrode. Wiley and Webster ${ }^{34}$ provided an analytical solution of current density distribution on the surface of a circular metal disc electrode showing a highly non-uniform distribution with the highest current density at the periphery. As one of the possible practical implementations of this condition, they suggested a segmented circular disc electrode by dividing the solid metal disc into many annular rings with a small solid disc at the center. Kim and Schimpf, ${ }^{13}$ Papazov et al.'26 Krasteva and Papazov17 also suggested that segmenting the electrode into concentric rings will reduce the edge effect and make a more uniform current density distribution. A study by Van Oosterom and Strackee ${ }^{23}$ evaluated the lead field from stimulation with bipolar concentric ring electrodes (CREs) and disc electrodes. The lead field is the electric field produced by stimulation from a reciprocally charged electrode. Their research suggested that CREs have a greater sensitivity directly under the electrode than disc electrodes.

Besio et al. ${ }^{6}$ found that noninvasive electrical stimulation, applied $5 \mathrm{~min}$ after status epilepticus onset via novel CREs on the scalp, reduced or abolished electrographic and behavioral seizure activity in pilocarpine-induced status epilepticus in rats. It may be possible that there are other applications where stimulation via CREs may have beneficial therapeutic properties. Further analysis is necessary to quantify stimulation characteristics such as current intensity, pulse duration, and pulse repetition rate parameters to determine the safety of electrical stimulation via CREs.

There are various types of noninvasive electrical stimulation that are approved by the Federal Drug Administration (FDA) as safe. One such form of transcranial electrical stimulation (TES) is electroconvulsive therapy (ECT), used to treat depression, which applies stimulation pulses up to $1000 \mathrm{~mA} .{ }^{29}$ Pacelat et al. reports on using up to $300 \mathrm{~mA}$ TES for intraoperative neurophysiology monitoring.25 Transcutaneous electrical nerve stimulation (TENS), frequently used for blocking pain, employs currents typically up to $30 \mathrm{~mA}$. 7 Transcranial direct current stimulation (tDCS), which has shown promise in epilepsy, is below $5.0 \mathrm{~mA} .10$ For transthoracic defibrillation (cardioversion), the Advancement of Medical Instrumentation (AAMI) specification for cardiac defibrillator devices sets the peak currents at $80,000 \mathrm{~mA}$ for a $25 \Omega$ load. Kerber et al. tested the efficacy of different currents up to 40,000 mA and impedances on cardioversion. ${ }^{12}$ In our epilepsy experiments we used 50 or $60 \mathrm{~mA} .{ }^{6}$ For the tissue analysis of this paper, we kept the TcES constant at $50 \mathrm{~mA}$.

In this study we quantitatively analyzed the effects of acute noninvasive electrical stimulation using CREs and determined the maximum safe current limit. We determined the relative energy density factor $J^{2} t$ introduced by Pearce et al. ${ }^{28}$ which is proportional to the delivered energy and is a measure of skin heating. A three-dimensional multi-layer finite element model was developed to perform an electrical-thermal coupled field analysis to calculate the temperature profile under the CRE and the corresponding energy density. In order to verify the results from the computer simulations, we performed animal experiments stimulating rats on the skin with the parameters of the simulation. The skin temperature was measured by infrared thermography. Controlled morphological studies using standard histological techniques to pathologically characterize any resulting tissue damage were also performed. 


\section{METHODS}

\section{Computer Model}

To understand what stimulation parameters would be safe using CREs, we first performed computer modeling and then physical experiments. The computer models described in this research were finite element models created with FEMLAB (Comsol, Inc., Birmingham, MA). We used the FEMLAB pre-coupled 'Joulean Heating' template to perform an electricalthermal coupled field analysis with temperature dependent properties.

We developed a simplified three-dimensional multilayer cylindrical model. Figure 1 shows a cross section of the three-dimensional model. The top layer was modeled as electrode-pluspaste, followed by the epidermis-plus-dermis layer, and the subcutaneous region, respectively. The $1.0 \mathrm{~cm}$ diameter CREs used in the study had an inner/outer ring radius of $4.6 / 5.1 \mathrm{~mm}$ and the inner disc had a diameter of $1.2 \mathrm{~mm}$. The CREs were modeled as a layer of $0.0 \mathrm{~mm}$ thickness followed by a $1.0 \mathrm{~mm}$ thick conductive paste layer. The epidermis and the dermis were modeled as one layer. In Fig. 1 the dimensions of each layer are specified in parenthesis. The dimensions of the overall model were $15 \mathrm{~mm}$ radius by $20 \mathrm{~mm}$ height. We performed simulations by supplying 50,75, and $100 \mathrm{~mA}$, respectively, to the outer ring. The disc was grounded in each simulation. All combinations of frequencies $100,250,350,500$, and $750 \mathrm{~Hz}$ and pulse-widths $50,100,150,200,250$, and $300 \mu$ s were used for each of the stimulation currents.

Boundary Conditions-The electrical and thermal boundary conditions used for the model are listed in Table 1. We applied input current pulses with current density $\left(J_{n}\right)$ at the outer ring of the CRE such that $n \cdot J=J_{n}$; where $n$ is the unit vector normal to the surface, $J$ is the inward current density vector, and $J_{n}$ is the normal component of the applied current density. The center disc served as an electrical ground return $(V=0)$. An electrical insulating boundary condition was applied to all the outer boundaries of the model such that $n \cdot J=0$; where $n$ is the unit vector normal to the surface and $J$ is the inward current density vector. All the internal boundaries at the interface of two layers were assigned continuity boundary condition $n \cdot\left(J_{1}\right.$ $\left.-J_{2}\right)=0$, where $J_{1}$ and $J_{2}$ are current densities in the neighboring layers such that the normal component of current was continuous across the internal boundaries.

We applied a thermal boundary condition of $T=T_{0}$ to the outermost boundary of the subcutaneous layer to simulate constant body core temperature at all times. A convective heat flux boundary condition $n \cdot(k \nabla T)=h\left(T_{\infty}-T\right)$ was specified at the upper epidermis and the conductive paste in contact with the environment. The epidermis convected heat into the surrounding environment at temperature $T \infty$ with heat transfer coefficient $h=5\left(\mathrm{~W} / \mathrm{m}^{2}{ }^{\circ} \mathrm{C}\right)$ and the conductive gel convected heat with heat transfer coefficient $h=10\left(\mathrm{~W} / \mathrm{m}^{2}{ }^{\circ} \mathrm{C}\right)$. All the internal boundaries at the interface of two layers were assigned continuity boundary conditions $n \cdot\left(q_{1}-q_{2}\right)=0$, where $q_{1}$ and $q_{2}$ are heat flux in the neighboring layers such that the normal component of heat flux was continuous across the internal boundaries. All the other surfaces of the model were assigned a thermal insulating boundary condition such that $n \cdot(k \nabla T)=0$. We assumed the initial potential distribution throughout the model to be constant $(V=0)$ and set the initial temperature of each layer to the body core temperature $\left(T=33^{\circ} \mathrm{C}\right)$. We applied biphasic charge balanced current pulses to the outer ring of the CRE.

\section{Experimental Verification}

To verify the results from the computer simulations, we performed experiments stimulating rats using the parameters of the computer simulations. The skin temperature distribution was measured using a high-speed detector thermography camera (60 frames per second 320X240, NEC TS300, Micro Health Systems, FL, USA). We used Sprague-Dawley rats. They were 
housed in shoe-box cages on a $12 \mathrm{~h}$ light/dark cycle. Our protocol was approved by the Louisiana Tech University IACUC.

Electrical Stimulation-We anesthetized all the animals $(n=7)$ with ketamine $(80 \mathrm{mg} / \mathrm{kg}$, $50 \mathrm{mg} / \mathrm{mL}$, i.p.) and xylazine $(12 \mathrm{mg} / \mathrm{kg}, 100 \mathrm{mg} / \mathrm{mL}$, i.p.) combination. Once they were anesthetized, we shaved the scalp and the back for the placement of the CREs. The rat skin was prepared using NuPrep ${ }^{\mathrm{TM}}$ (Weaver \& Co., Aurora, CO) abrasive gel. The CREs were coated with high conductivity Ten $20^{\mathrm{TM}}$ electrode paste (Weaver \& Co., Aurora, $\mathrm{CO}$ ) to adhere the CRE to the skin and reduce the electrode-skin impedance. We used a template to wipe the CRE-paste combination and provide a smooth $1.0 \mathrm{~mm}$ thick layer of Ten $20^{\mathrm{TM}}$ electrode paste. Then we placed the CRE-paste combination on the skin of the rat for the experiment. The CRE paste combination was allowed to equilibrate with the skin. We found that it took approximately $5 \mathrm{~min}$ for the equilibrium, but we waited $15 \mathrm{~min}$. Five or six experiments with different current, frequency, pulse width combinations were preformed on each animal at different locations. We applied each stimulation for a period of $60 \mathrm{~s}$. After the stimulation, we removed the CREs and then prepared the stimulation site for histological analysis as described next.

The stimulation pulses were constant current symmetric biphasic charge-balanced square pulses. For verification of proper stimulation, we recorded the voltage impressed by the stimulation pulses across a one Ohm resistor. At the end of the experiments, we euthanized the animals and histologically examined the skin to determine the extent of tissue damage.

\section{Histological Analysis}

We performed tissue fixation by transcardial perfusion with $10 \%$ neutral buffered formalin of the deeply anesthetized animal. Skin samples, approximately $15 \times 15 \mathrm{~mm}$ square, centered on each of the five or six electrode positions were removed and placed in $10 \%$ formalin to complete the fixation. We bisected each sample through the center of the stimulation site. Then we dehydrated the samples by immersing the tissue in a series of solutions of ethyl alcohol and water, with gradually increasing percentage of $(75,95$, and $100 \%)$ alcohol. Next we cleared (Histoclear ${ }^{\mathrm{TM}}$ ) the tissue and embedded it in paraffin. We cut sections at $10 \mu \mathrm{m}$, stained them with hematoxylin and eosin and viewed them with bright-field microscopy or polarizing microscopy. Hematoxylin is a basic stain which has affinity for acidic substances such as DNA, RNA, etc. It therefore stains nuclei, ribosomes and cartilage matrix bluish violet, and these structures are referred to as 'basophilic'. Eosin is an acid stain which has affinity for basic substances such as protein. It stains almost everything red except DNA and RNA. Images were made and are provided in the "Results" section.

\section{RESULTS}

As an initial verification of the computer models, we simulated the physical animal experiments of Pearce $e t a l .{ }^{28}$ in the form of three-dimensional finite element models. These models were built according to Pearce $e t a l .{ }^{28}$ specified experimental conditions. Separate models were made for each stimulation parameter combination experiment. A paired two-sided $t$-test showed no statistically significant difference between the computer models and the results reported by Pearce for the maximum temperatures at low energy ( $p=0.082$ left, 0.142 right) and medium energy ( $p=0.068$ left, 0.082 right) density stimulations, respectively, but showed a statistically significant difference ( $p=0.011$ left, 0.037 right) for high energy density stimulations.

\section{Simulation Results for CRE}

We compared the maximum temperatures resulting from the simulated electrical stimulation with different energy densities. The three-dimensional multilayer cylindrical models described 
previously were implemented to generate these results. The simulated maximum temperature at the skin surface for energy density factor $J^{2} t<.92\left(\mathrm{~A}^{2} / \mathrm{cm}^{4} \cdot \mathrm{s}^{-1}\right)$ beneath the $1.0 \mathrm{~cm} \mathrm{CRE}$ never exceeded $45^{\circ} \mathrm{C}$ which is the threshold temperature for skin damage. For energy density factor $J^{2} t=0.92-1.5\left(\mathrm{~A}^{2} / \mathrm{cm}^{4} \cdot \mathrm{s}^{-1}\right)$, the maximum simulated skin temperature below the CRE was between 45 and $56^{\circ} \mathrm{C}$. For energy density factor $J^{2} t>1.5\left(\mathrm{~A}^{2} / \mathrm{cm}^{4} \cdot \mathrm{s}^{-1}\right)$, the skin temperature was greater than $56^{\circ} \mathrm{C}$.

\section{Results of the Experimental Verification}

The objective of the animal experiments was to measure the maximum temperature from different stimulation parameters applied to the skin surface of rats to verify our simulation results. The stimulation parameters included those thought to be necessary for humans. This is based on estimates of the current density thresholds for stimulation of peripheral nerve ${ }^{21}$ and of the motor cortex ${ }^{16}$ using magnetic stimulation yielding rheobase values of approximately $2.5 \mathrm{~A} / \mathrm{m}^{2}$. According to a separate finite element model, not reported on here, we can achieve $2.5 \mathrm{~A} / \mathrm{m}^{2}$ approximately $1.0 \mathrm{~cm}$ deep into the cortex of the human brain with $50 \mathrm{~mA}$ stimulation from a $1.0 \mathrm{~cm}$ CRE. We used infrared thermography to measure the temperature distribution under and around the CRE while electrical stimulation was applied. We studied two different sets of stimulation parameters: a stimulus of $50 \mathrm{~mA}, 200 \mathrm{~Hz}, 300 \mu \mathrm{s}$ - low energy density $\left(J^{2} t=0.7\right)\left(\mathrm{A}^{2} / \mathrm{cm}^{4} \cdot \mathrm{s}^{-1}\right)$, and a stimulus of $50 \mathrm{~mA}, 500 \mathrm{~Hz}, 300 \mu \mathrm{s}-$ high energy density $\left(J^{2} t=2.7\right)\left(\mathrm{A}^{2} / \mathrm{cm}^{4} \cdot \mathrm{s}^{-1}\right)$. Temperature profiles for low energy density factor were recorded after the CREs were placed prior to stimulation (Fig. 2a), $1 \mathrm{~s}$ after the stimulation was turned ON (Fig. 2b), $30 \mathrm{~s}$ into the stimulation (Fig. 2c), and at the end of stimulation (60 s) (Fig. 2d), respectively. Immediately after the stimulation, we removed the CREs and recorded the temperature profile of the skin surface (Fig. 2e) and the CRE surface (Fig. 2f). As seen in Fig. 2, the temperature distribution under the CRE is non-uniform and the maximum temperature was observed under the disc. These observations were in agreement with the results from simulations of the models.

The maximum temperature measured from the rat experiments using low energy density stimulations was $38^{\circ} \mathrm{C}$. This result is very similar to the simulation result, which was $39^{\circ} \mathrm{C}$ for low energy density stimulation. The maximum temperature measured from the experiments using high energy density stimulations was $47^{\circ} \mathrm{C}$. The maximum temperature from simulations for high energy density was nearly $78^{\circ} \mathrm{C}$.

\section{Results of the Histological Analysis}

We examined tissue from three different energy density factors for evidence of histopathology. The following pathological changes were noted (Table 2): (1) Epidermal necrosis as indicated by changes in density, compaction, and/or nuclei; (2) Damage to sebaceous glands and hair follicles as indicated by changes in density, compaction, and/or nuclei; and (3) Damage in the dermis as indicated by changes in density and orientation of collagen fiber bundles.

A section of un-stimulated skin is shown in Fig. 3a. The epidermis is intact, with moderately stained cytoplasm and lightly stained circular nuclei. The collagen fibers, although not shown in this section, are evenly distributed and in small bundles.

Stimulation at the low energy density factor $J^{2} t<0.92\left(\mathrm{~A}^{2} / \mathrm{cm}^{4} \cdot \mathrm{s}^{-1}\right)$ showed little or no damage (Fig. 3b). The basal nuclei of the epidermis were more darkly stained. The medium energy density factor $J^{2} t=0.92-1.5\left(\mathrm{~A}^{2} / \mathrm{cm}^{4} \cdot \mathrm{s}^{-1}\right)$ showed some moderate changes (Fig. 3c). The epidermal cells were less distinct at most of the stimulation sites. The nuclei were shrunken darkly stained and sometimes indistinct. The thickness of the epidermis appeared thinner than in the controls. We also observed damage to the hair follicles and sebaceous glands. High energy density factor stimulation $J^{2} t>-1.5\left(\mathrm{~A}^{2} / \mathrm{cm}^{4} \cdot \mathrm{s}^{-1}\right)$ showed more pronounced damage to 
the epidermis (Fig. 3d). The epidermis was compact and homogenously stained and no nuclei were present. The cells in the deeper epidermis were indistinct with darkly stained elongated nuclei. Collagen fibers in the dermis were clumped and different in orientation compared with the control tissue.

\section{DISCUSSION}

The objective of this study was to quantitatively analyze the effects of acute noninvasive electrical stimulation using CREs and determine the maximum safe current limit. We made this determination by computer modeling and physical experiments.

We developed a three-dimensional multilayer model to determine the temperature profile under the CRE using FEMLAB. The FEMLAB pre-coupled 'Joulean Heating' template was used to perform an electricalthermal coupled field analysis with temperature dependent properties. Since heat transfer between arterial blood and the surrounding tissue should occur only in the deep dermis, unknown and uncontrolled parameters such as blood perfusion and metabolic activity were not modeled to simplify the heat transfer equation. As a further simplification, we assumed that the conductive paste was a perfect conductor and there was perfect contact between skin and the CRE. The current could not enter or leave the model except at the active and ground electrodes. All the outer boundaries were electrically and thermally insulated. We assumed the electrical and thermal properties of all the layers to be homogenous, isotropic and independent of temperature to minimize the mathematical complexity of the differential equations. It was shown that the inclusion of electrical conductivity values for both grey and white brain matter unnecessarily increased the complexity of the model. ${ }^{9}$ Although they did not calculate the temperatures they reported that induced electric fields in models with homogenous brain tissue varied slightly from the electric fields in models with a heterogeneous brain layer, and were therefore more efficient. All of the outer surfaces of the model were assumed to be adiabatic and the initial temperature distributions within the conductive paste and tissue layers were uniform at $T=33^{\circ} \mathrm{C}$.

Despite these extensive simplifications, the reproduction of Pearce et al. ${ }^{28}$ experimental results correlating the maximum temperature and safe level of electrical stimulation suggests that the simulation results provide a good estimate of the tissue temperature comparable to that obtained from the animal experiments. The resultant maximum temperatures from the simulations match those from Pearce et al. ${ }^{28}$ experiments except at the higher energy density factors. There was no statistically significant difference between the computer models and the results reported by Pearce $e t a l$. for the maximum temperatures of low and medium energy density stimulations but a statistically significant difference existed for high energy density stimulations.

The statistically significant difference in the results for high energy density stimulations may be explained by the increasing electrical conductivity of conductive paste and tissue with increasing temperature. High energy density stimulations result in very high temperatures under the CREs which may lead to unpredictably large changes in the thermal and electrical properties of both tissue and the electrode paste which cannot be accounted for in the simplified models used for this study.

In order to verify the results from the computer simulation, we performed physical experiments stimulating rats using the parameters of the simulation. The temperature distribution on the skin was measured using infrared thermography. The infrared thermographs closely corresponded to those predicted by the simulations, showing a non-uniform temperature distribution under the CRE with maximum temperature observed under the disc. The maximum temperature measured from the thermographs for Low energy density stimulations was $38^{\circ} \mathrm{C}$ and was very close to the $39^{\circ} \mathrm{C}$ of the simulation results for low energy density stimulation. 
The maximum temperature measured from the experiments for high energy density stimulations was $47^{\circ} \mathrm{C}$ and the maximum temperature from simulations for high energy density was nearly $78^{\circ} \mathrm{C}$. This gap may be due to the fact that the melting point of the conductive paste was $43{ }^{\circ} \mathrm{C}$. As the temperature increases above $43^{\circ} \mathrm{C}$, the paste starts melting and its electrical and thermal conductivity increases, resulting in a decrease in the maximum temperature. It could also relate to the actual thermal properties of vascular tissue which was not simulated.

A possible limitation of the study was that we tested the stimulation on rat skin rather than human skin. Rat scalp/skin is commonly used as a skin model instead of testing on humans. ${ }^{2}$ We shaved the rat scalp and skin to apply the electrode Ten $20^{\mathrm{TM}}$ paste combination. This was necessitated by the dense coat of hair that rats have. Fortunately human hair is not as dense as rat hair. We have recorded various evoked potential electroencephalography (EEG) signals from humans without having to shave their heads. ${ }^{4,5}, 15 \mathrm{We}$ simply use a mild abrasive and then apply a layer of Ten $20 \mathrm{TM}$ paste, approximately $1.0 \mathrm{~mm}$ thick, using a template.

Conceptually, when applying the stimulation to humans without shaving the scalp, any hair under the electrode may act as a thermal insulator blocking heat from transferring from the electrode through the paste to the scalp or skin.

In order to determine if damage occurred as a result of the stimulation, we examined the tissue in the areas of the stimulation events microscopically. Low energy density showed minor changes in the upper layers of the epidermis where the CRE electrode made contact. The dermis appeared normal when observed with bright-field microscopy. Polarizing microscopy of stained tissue showed a slight loss in birefringence compared to the controls, not shown. The damage to the cytoplasm and nuclei were consistent with reversible changes. The medium and high energy tissues showed more damages and are less likely to be reversible.

\section{CONCLUSIONS}

We conclude from this study that the simulation results of the three-dimensional models are accurate for low energy density distributions. It can also be concluded that as long as the specified energy density applied through the CRE is kept below $0.92\left(\mathrm{~A}^{2} / \mathrm{cm}^{4} \cdot \mathrm{s}^{-1}\right)$, the maximum temperature will remain within the safe limits and also within the limits of the melting point of conductive paste and provide a safe current density distribution. Further, there are no specifications on the conductivity of the Ten $20^{\mathrm{TM}}$ electrode paste. Future work should focus on the effects of the conductivity and thickness of the electrode paste.

\section{Acknowledgments}

The authors would like to thank Green Family Chiropractic of Farmerville Louisiana for the use of there infrared thermography system and Dr. Mesut Sahin for rat experimental training and use of his laboratory.

\section{REFERENCES}

1. Ambler JJ, Sado DM, Zideman DA, Deakin CD. The incidence and severity of cutaneous burns following external DC cardioversion. Resuscitation 2004;61:281-288. [PubMed: 15172706]

2. Auletta C. Current in vivo assays for cutaneous toxicity: local and systemic toxicity testing. Basic Clin. Pharmacol. Toxicol 2004;95:201-208. [PubMed: 15546473]

3. Balmaseda MT Jr, Fatehi MT, Koozekanani SH, Sheppard JS. Burns in functional electric stimulation: two case reports. Arch. Phys. Med. Rehabil 1987;68:452-453. [PubMed: 3496867]

4. Besio W, Cao H, Zhou P. Application of tripolar concentric electrodes and pre-feature selection algorithm for brain-computer interface. IEEE Trans. Neural Syst. Rehabil. Eng 2008;16(2):191-194. [PubMed: 18403288] 
5. Besio W, Koka K, Aakula R, Dai W. Tri-polar concentric electrode development for high resolution EEG Laplacian electroencephalography using tri-polar concentric ring electrodes. IEEE Trans. BME 2006;53(5):926-933.

6. Besio W, Koka K, Cole A. Effects of noninvasive transcutaneous electrical stimulation via concentric ring electrodes on pilocarpine-induced status epilepticus in rats. Epilepsia 2007;48(12):2273-2279. [PubMed: 17651415]

7. Cowan S, McKenna J, McCrum-Gardner E, Johnson M, Sluka K, Walsh D. An investigation of the hypoalgesic effects of TENS delivered by a glove electrode. J. Pain 2009;10:694-701. [PubMed: 19398378]

8. Danielsen L, Gniadecka M, Thomsen HK, Pedersen F, Strange S, Nielsen KG, Petersen HD. Skin changes following defibrillation. The effect of high voltage direct current. Forensic Sci. Int 2003;134:134-141. [PubMed: 12850408]

9. Davey K, Epstein C, George M, Bohning D. Measuring the effects of electrical conductivity of the head on the induced electric field in the brain during magnetic stimulation. Clin. Neurophys 2003;114 (11):2204-2209.

10. Fregni F, Thome-Souza S, Nitsche M, Freedman S, Valente K, Pascual-Leone A. A controlled clinical trial of cathodal DC polarization in patients with refractory epilepsy. Epilepsia 2006;47:335-342. [PubMed: 16499758]

11. Grossi EA, Parish MA, Kralik MR, Glassman LR, Esposito RA, Ribakove GH, Galloway AC, Colvin SB. Direct-current injury from external pacemaker results in tissue electrolysis. Ann. Thorac. Surg 1993;56:156-157. [PubMed: 8328848]

12. Kerber R, Kieso R, Kienzle M, Olshansky B, Waldo A, Carlson M, Wilber D, Aschoff A, Birger S, Charbonnier F. Current-based transthoracic defibrillation. Am. J. Cardiol 1996;78:1113-1118. [PubMed: 8914873]

13. Kim Y, Schimpf PH. Electrical behavior of defibrillation and pacing electrodes. Proc. IEEE 1996;84:446-456.

14. Kim Y, Webster JG, Tompkins WJ. Simulated and experimental studies of temperature elevation around electrosurgical dispersive electrodes. IEEE Trans. Biomed. Eng 1984;31:681-692. [PubMed: 6500588]

15. Koka K, Besio W. Improvement of spatial selectivity and decrease of mutual information of tri-polar concentric ring electrodes. J. Neurosci. Methods 2007;165:216-222. [PubMed: 17681379]

16. Kowalski T, Silny J, Buchner H. Current density threshold for the stimulation of neurons in the motor cortex area. Bioelectromagnetics 2002;23(6):421-428. [PubMed: 12210560]

17. Krasteva VT, Papazov SP. Estimation of current density distribution under electrodes for external defibrillation. Biomed. Eng. Online 2002;16:1-7.

18. Lambert, H.; De Baetselier, E.; Vanalme, G.; Mey, GD. Skin burn risk using transcutaneous direct current; Proceedings of IEEE Engineering in Medicine and Biology 17th Annual Conference; 1995. p. 477-478.

19. Lippmann M, Fields WA. Burns of the skin caused by a peripheral-nerve stimulator. Anesthesiology 1974;40:82-84. [PubMed: 4810323]

20. Merrill D, Bikson M, Jefferys J. Electrical stimulation of excitable tissue: design of efficacious and safe protocols. J. Neurosci. Methods 2005;141:171-198. [PubMed: 15661300]

21. Miranda PC, Lomarev M, Hallett M. Modeling the current distribution during transcranial direct current stimulation. Clin. Neurophysiol 2006;117(7):1623-1629. [PubMed: 16762592]

22. Moritz R, Henriques FC. Studies of thermal injury: II. The relative importance of time and surface temperature in the causation of skin burns. Am. J. Pathol 1947;23:695-720. [PubMed: 19970955]

23. Oosterom AV, Strackee J. Computing the lead field of electrodes with axial symmetry. Med. Biol. Eng. Comput 1983;21:473-481. [PubMed: 6888015]

24. Overmeyer KM, Pearce JA, DeWitt DP. Measurements of temperature distributions at electrosurgical dispersive electrode sites. J. Biomech. Eng 1979;101:66-72.

25. Pacelat E, Magjarevic R, Isgum V. Measurement of electrode-tissue interface characteristics during high current transcranial pulse electrical stimulation. Measurement 2000;27:133-143.

26. Papazov S, Kostov Z, Daskalov I. Electrical current distribution under transthoracic defibrillation and pacing electrodes. J. Med. Eng. Technol 2002;26:22-27. [PubMed: 11924843] 
27. Patriciu A, Yoshida K, Struijk JJ, DeMonte TP, Joy ML, Stodkilde-Jorgensen H. Current density imaging and electrically induced skin burns under surface electrodes. IEEE Trans. Biomed. Eng 2005;52:2024-2031. [PubMed: 16366226]

28. Pearce JA, Geddes LA, Van Vleet JF, Foster K, Allen J. Skin burns from electrosurgical current. Med. Instrum 1983;17:225-231. [PubMed: 6877129]

29. Sackeim H. Convulsant and anticonvulsant properties of ECT towards a focal form of brain stimulation. Clin. Neurosci. Res 2004;4:39-57.

30. Shah, RN.; Webster, JG. Burns under electrosurgical dispersive electrodes; Proceedings of the 14th Annual Meeting AAMI; Las Vagas, NV: 1979. p. 292

31. Stoner DL, Yoo JH, Feldtman RW, Stanford W. Human skin burns induced by defibrillator default current. J. Thorac. Cardiovasc. Surg 1976;72:157-161. [PubMed: 933550]

32. Takamiya M, Saigusa K, Nakayashiki N, Aoki Y. A histological study on the mechanism of epidermal nuclear elongation in electrical and burn injuries. Int. J. Legal Med 2001;115:152-157. [PubMed: 11775017]

33. Vedovato JW, Polvora VP, Leonardi DF. Burns as a complication of the use of diathermy. J. Burn Care Rehabil 2004;25:120-123. [PubMed: 14726750]

34. Wiley JD, Webster JG. Analysis and control of the current distribution under circular dispersive electrodes. IEEE Trans. Biomed. Eng 1982;29:381-385. [PubMed: 7084970] 


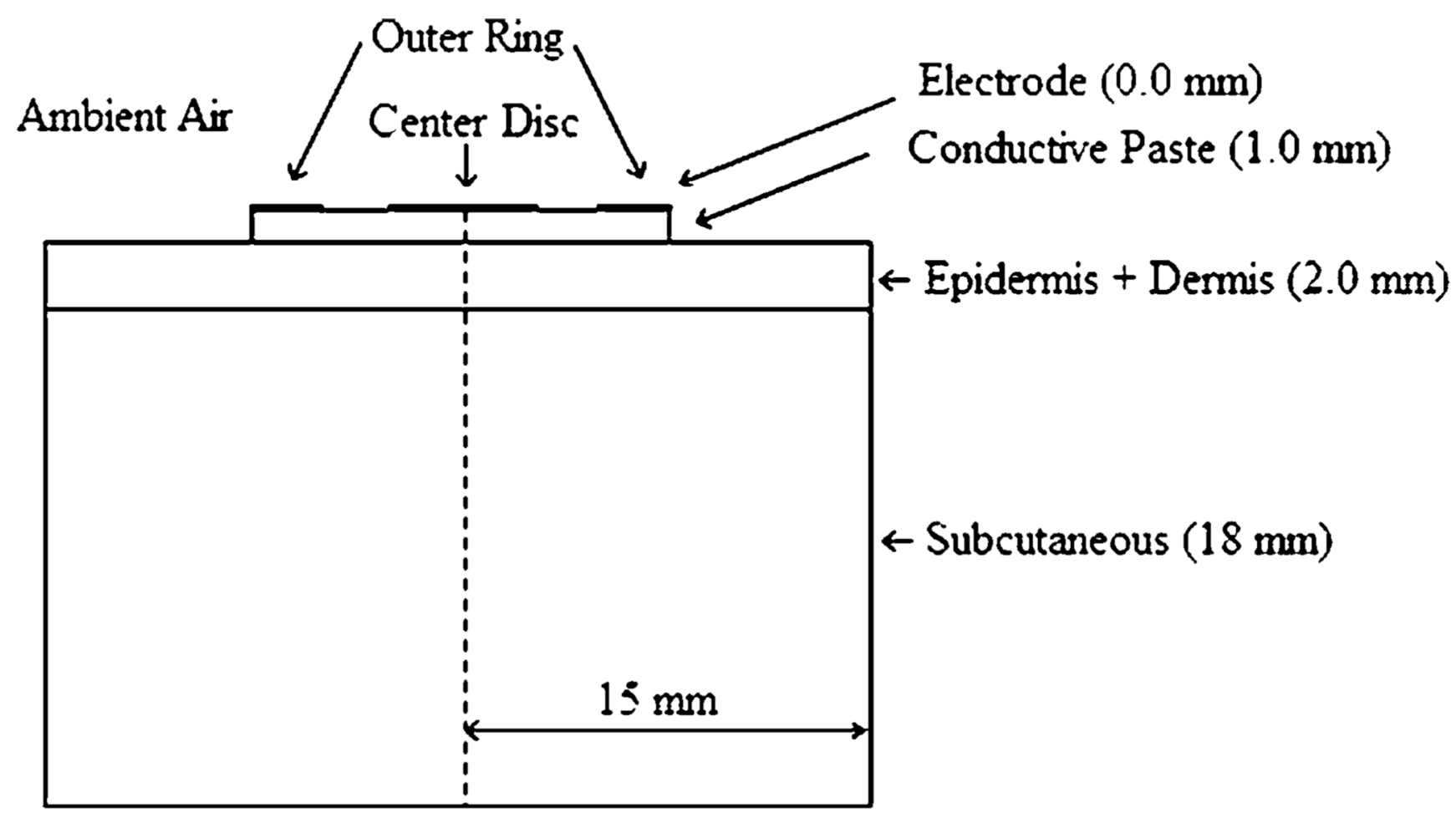

FIGURE 1.

The schematic representation of the finite element computer model showing the electrode and conductive paste on top of the tissue. 
(a)

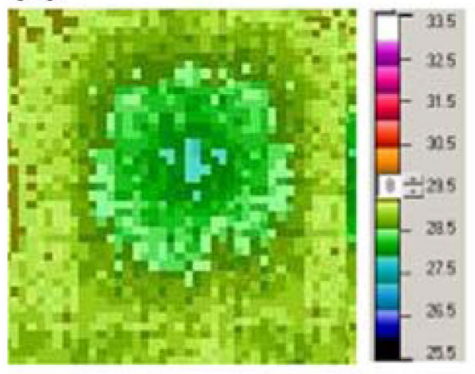

Initial Temp. profile

(d)

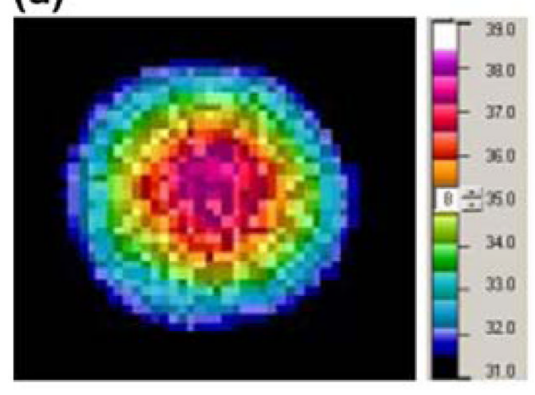

Temp. at the end of stimulation (b)
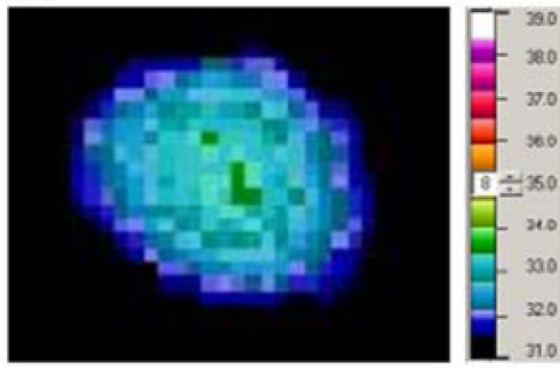

Temp. at the begining of stimulation

(e)

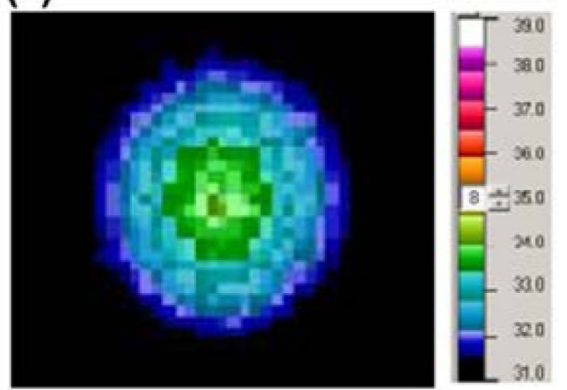

Temp. at the skin surface after removing the electrode (c)

Temperatures in ${ }^{\circ} \mathrm{C}$

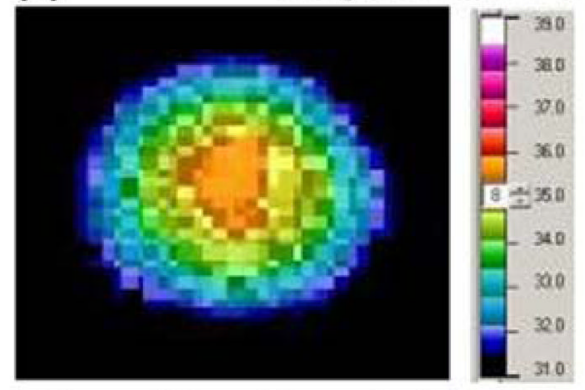

Temp. $30 \mathrm{sec}$ into stimulation

(f)

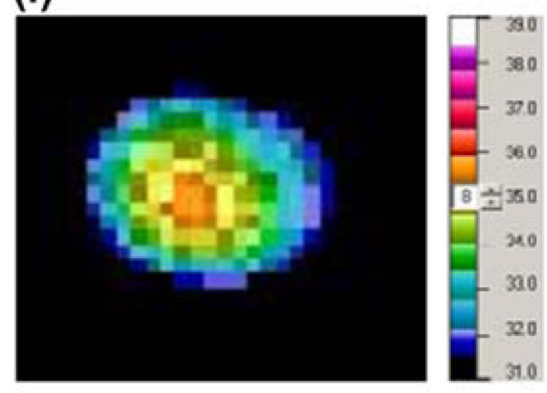

Temp. at the sufrace of the electrode

FIGURE 2.

Temperature profiles for low energy density factor stimulation $\left(J^{2} t=0.7\right)\left(\mathrm{A}^{2} / \mathrm{cm}^{4} \cdot \mathrm{s}^{-1}\right)$. The panel (a) temperature scale is from 25.5 to $33.5^{\circ} \mathrm{C}$ while all other panels are from 31.0 to 39.0 ${ }^{\circ} \mathrm{C}$. 

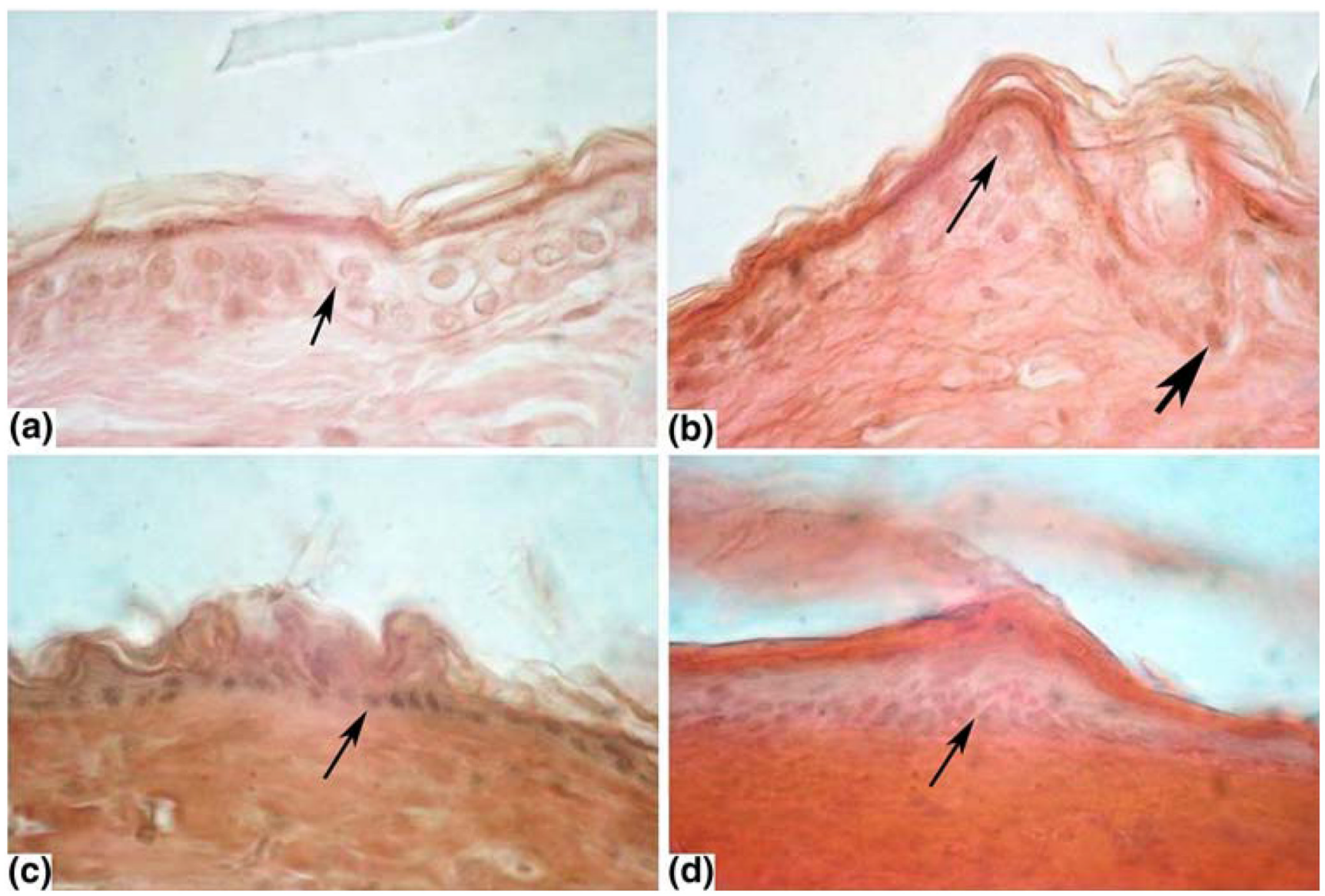

FIGURE 3.

Selected microscopic images: Initial magnification of 40×. (a) Control: cells appear distinct with nuclei visible. (b) Low energy: most nuclei appear normal (thin arrow). Some are more densely stained (bold arrow). (c) Medium energy: epidermis is more compact and most nuclei are more densely stained (arrow). (d) High energy: cytoplasm is densely stained and nuclei are shrunken, densely stained or missing above the germinal area. Nuclei in the germinal area are dense and elongated. 


\section{TABLE 1}

Boundary conditions.

\begin{tabular}{lll}
\hline Type & Boundary & Expression \\
\hline DC conductive media module & & \\
Inward current flow & Outer ring & $n \cdot J=J_{n}$ \\
Ground & Center disc & $V=0$ \\
Electrical insulation & All remaining boundaries & $n \cdot J=0$ \\
Continuity & All internal boundaries & $n \cdot\left(J_{1}-J_{2}\right)=0$ \\
Heat transfer module & & \\
Convective heat flux & Epidermis, conductive paste & $n \cdot(k \nabla T)=h\left(T_{\infty}-T\right)$ \\
Prescribed temperature & Subcutaneous & $T=T_{0}$ \\
Thermal insulation & All remaining boundaries & $n \cdot(k \nabla T)=0$ \\
Continuity & All internal boundaries & $n \cdot\left(q_{1}-q_{2}\right)=0$ \\
\hline
\end{tabular}




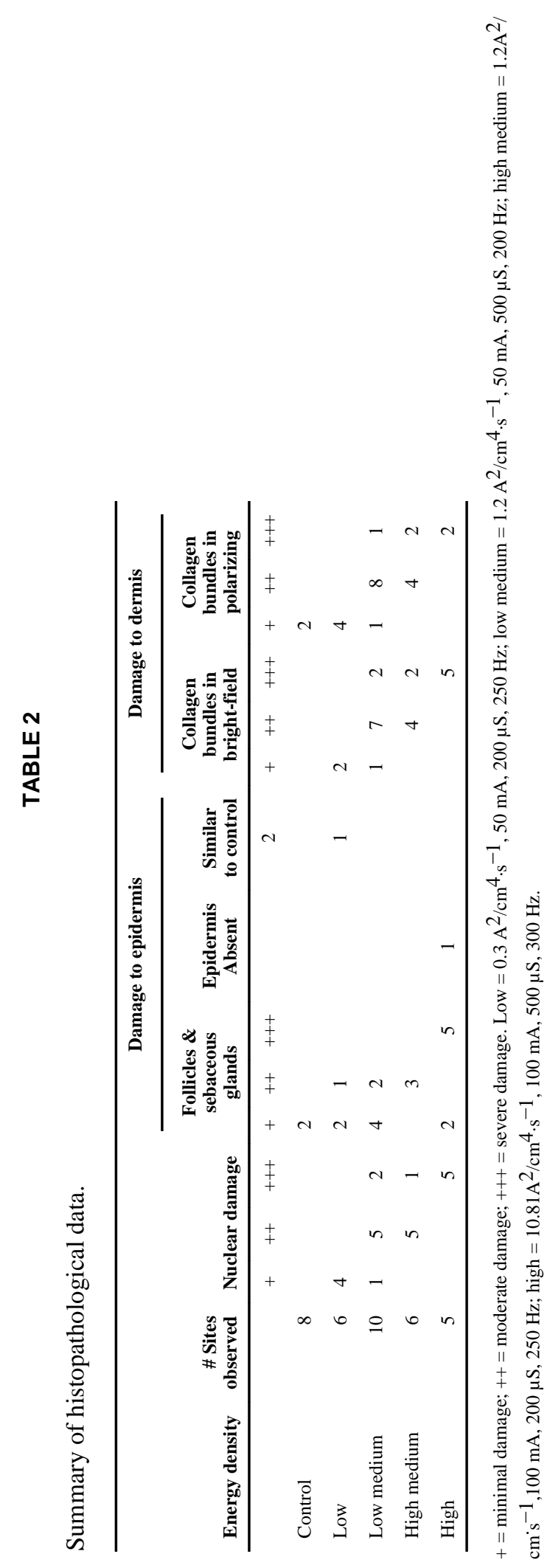

Ann Biomed Eng. Author manuscript; available in PMC 2011 March 1. 